\title{
Logistik, Lagerhaltung und Bestellwesen
}

\author{
Matthias Müller, Andreas Luther, Petra Ertmer, Karl Heinrich Winker
}

\section{Zusammenfassung}

Die gebotene effektive Nutzung der Ressourcen gerade im DRG-Zeitalter macht die Optimierung verschiedener Prozesse im OP, als einem der kostenintensivsten Bereiche des Krankenhauses, notwendig. Um bei der Veränderung der OP-Logistik mit Lagerhaltung und Bestellwesen die Ansprüche der Patienten, des OP-Personals und des Betriebswirtschaftlers zu erfüllen, sind genaue Analysen des aktuellen Zustandes, des Bedarfes und des Standardisierungsgrades notwendig. Die Grundvoraussetzung für die dann notwendige Reorganisation der OP-Ab- läufe ist wegen ihrer nahezu unüberschaubaren Komplexität und der dafür notwendigen Materialien eine frühzeitige und engste Einbeziehung des Fachpersonals. Ohne die Fachkenntnis des OP-Personals (Ärzte und der OPSchwestern) ist eine sinnvolle Neustrukturierung der OP-Logistik nicht durchführbar und würde weit reichende negative Folgen für die Patientenversorgung haben. Eine konsequente Weiterentwicklung der Optimierung der OP-Logistik wird perspektivisch eine vollständige elektronische Beschaffung (E-Procurement) sein. de Information den OP-Schwestern (und -pflegern) ${ }^{1}$ vorliegt (gewünschte Implantate, Alternativverfahren, zu operierende Seite und Metallallergie), stellen diese die benötigten Materialien auf einem Fallwagen zusammen.

Diese Materialien lassen sich wie folgt nach Herkunft und Art grob klassifizieren:

- Instrumente
Implantate
- Wäsche
- Unspezifische Verbrauchsmaterialien

Um dieses, bei erster Ansicht recht einfache System entwerfen und erfolgreich benutzen zu können, sind viele Überlegungen, Vorarbeiten und Voraussetzungen nötig.

Um einen medizinisch reibungslosen Ablauf für diese Operation zu gewährleisten, seien die Ansprüche der im OP tätigen Personen aufgezählt:

Auswahl und größeren Mengen, schnelle Nachlieferung bei unvorhersehbarem Mehrverbrauch, fehlerlos bestückte Siebe... Auf der anderen Seite stehen die betriebswirtschaftlichen Notwendigkeiten, nicht zuletzt durch die Einführung der DRG's: Kostenminimierung, Erlössteigerung, Leistungs- und Kostentransparenz.

Die Logistik, also die Gestaltung und Kontrolle des Material- und Informationsflusses und der integrierten Beschaffung, wurde lange Zeit in ihrer Bedeutung unterschätzt. Bei Sachkosten von 18 Milliarden Euro in deutschen Krankenhäusern sehen vorsichtige Schätzungen ein Einsparpotenzial von 7-10\% der Sachkosten bei Reorganisation des Einkaufs und von 20-25\% [1] im Prozesskostenbereich.

Ein Standardbeispiel aus unserer Klinik, auf das wir auch im weiteren Artikel eingehen werden, soll die komplexen Abläufe verdeutlichen:

Ein Patient mit einer Sprunggelenksfraktur soll durch innere Osteosynthese versorgt werden. Nachdem die entsprechen-

Die Operateure erwarten, dass die notwendigen Implantate und Instrumente in ausreichender Auswahl vorhanden sind und ohne Verzögerung und ohne technische Fehler angereicht werden. Eventuelle Komplikationen, wie während der Operation unbrauchbar gewordene Implantate oder die Notwendigkeit, auf ein anderes Implantat oder ein anderes Verfahren zu wechseln, müssen sofort beherrscht werden können.

Die Erwartungen der OP-Schwestern sind: Die Zusammenstellung des Fallwagens muss anhand der Operation transparent und bekannt sein, die Siebe müssen vollständig und technisch fehlerlos gepackt sein, die Verbrauchsmaterialien müssen schnell zugänglich sein, diese müssen in ausreichender Anzahl und

\footnotetext{
${ }^{1}$ Im Folgenden wird aus Übersichtlichkeitsund Lesbarkeitsgründen nur eine Geschlechtsform gewählt
} 
ohne abgelaufenes Haltbarkeitsdatum vorhanden sein.

Weiterhin muss bekannt sein, um bei oben genanntem Beispiel zu bleiben, wie viele Sprunggelenksfrakturen pro Tag operiert werden können und wie lange bis zur Versorgung der nächsten Sprunggelenksfrakturen gewartet werden muss. Wie sind die Zeiten in der Sterilisation und wie sind die Zeiten für die Lieferung der verbrauchten Osteosynthesematerialien?

Würde man eine Logistik und ein Bestellsystem allein nach den Wünschen der Anwender entwerfen, bedeutete dies einen großen Vorrat an Materialien, das OP-Personal würde sich um eine vorgezogene Endkontrolle kümmern und damit die neuralgischen Punkte der Bestellung (direkt nach Verbrauch der Platte würde das Lager durch eine direkte Bestellung beim Hersteller wieder aufgefüllt) und die Bestückung der Siebe in „fachspezifischer" Hand lassen. Das Wissen um diese komplexen Vorgänge müsste nicht transparent sein und die Logistik im OP würde durch lange Erfahrung und mündlich weiter gegebene und gesammelte Informationen organisiert sein.

Aus Sicht des Betriebswirtschaftlers ist ein solches Bestellsystem nicht akzeptabel. Aus seiner Sicht müssen die Kosten optimiert werden. Die Organisation der Logistik und damit des Bestellwesens durch das OP-Personal ist ein Reagieren auf direkten Verbrauch und ist wegen des Fehlens der fachlichen (in diesem Fall betriebswirtschaftlichen) Kompetenz nicht in der Lage, Einsparpotenziale (Synergieeffekte, Sammelbestellungen, Vergleichsangebote...) wahrzunehmen. Die im Haus vorgehaltenen Materialien verursachen Lagerungskosten und binden, da es sich um gekaufte Ware handelt, Kapital. Aus der Sicht des Wirtschaftlers ergeben sich Einsparmöglichkeiten und Optimierungen bei

- der Menge der vorzuhaltenden Ware (Produkte, Implantate)

- der Vielzahl der verwendeten Materialien (inklusive der Osteosynthesematerialien)

- der Delegation von Arbeiten an Mitarbeiter anderer Gehaltsgruppen

- der Standardisierung und Transparenz, damit der Wissensverlust durch Personalfluktuation oder -umsetzung minimiert wird
Genaueste Datenerhebung und Standardisierung sind die Voraussetzungen für eine Optimierung der OP-Logistik, insbesondere der vorzuhaltenen Ware (Implantate).

Die erste Voraussetzung für das betriebswirtschaftliche Eingreifen in diese sensiblen medizinischen Prozesse ist eine genaueste Datenerhebung. Nachdem die Operationshäufigkeiten und der Materialverbrauch vorliegen, erfolgt die Analyse:

- Von Artikelwert und Artikelmenge nach dem Umsatzvolumen (ABC-Analyse $^{2}[2]$ )

- Des Beschaffungs- und Ausfallrisikos

- Der Anforderung und Lieferung, die einen konstanten, einen saisonalen und stochastischen Verlauf zeigen (XYZ-Analyse ${ }^{3}$ )

Zur zweiten Voraussetzung kommen wir, weil trotz einer akribischen Auswertung guter Rohdaten das Ergebnis immer ein statistisches Ergebnis ist, im positiven wie im negativen Sinne. Natürlich ist es von außerordentlichem Interesse zu wissen, dass zum Beispiel 6 verschiedene Operationsverfahren $80 \%$ aller Eingriffe ausmachen und 6 verschiedene Verbrauchsartikel 85\% des Aufkommens aller Verbrauchsmaterialien abdecken [3]. Aber heißt das, dass mit vielleicht 20 Verbrauchsartikeln fast alle vorhanden sind und nur minimale Einbußen in Kauf genommen werden müssen? Kann man auf ein Implantat vollständig verzichten, weil es (bei unserer Sprunggelenksfraktur) winkelstabile Drittelrohrplatte heißt und nur wenige Mal im Jahr benötigt wird? Muss ich die doppelte Menge der benötigten Implantate vorhalten, weil die Platte, der Nagel oder der Fixateur interne seitenspezifisch sind oder muss ich die 4-fache Menge vorhalten, weil es auch Stahlausführungen gibt und die Wahrscheinlichkeit einer Chrom-Nickel-Allergie ca. $10 \%$ beträgt? Kann man statistisch gesehen über das Jahr eine 30\% Reserve besitzen, oben genannte Sprunggelenksfraktur intern zu stabilisieren, aber saisonal (in den Wintermonaten) die Hälfte aller Patienten auf eine Operation 5 Tage nach dem Unfall vertrösten? Und wie viele erfahrene Operateure einer Klinik kennen denn die Anzahl der im Haus ver-

\footnotetext{
${ }^{2}$ Die Kombination einer Wert- und einer Mengenstrukturanalyse wird ABC-Analyse genannt

${ }^{3}$ Die XYZ-Analyse klassifiziert Artikel nach ihrer Umsatzregelmäßigkeit beziehungsweise Verbrauchsschwankung
}

fügbaren Implantate (im Lager) und die Anzahl der speziellen Siebe und deren Bestückung und damit den Gesamtbestand?

Auch korrekte statistische Ergebnisse müssen durch Spezifika ergänzt werden (Erfahrung der Operateure und der OPSchwestern).

Deshalb heißt die zweite Voraussetzung für die betriebswirtschaftliche Optimierung der OP-Logistik:

Einbeziehung des Fachpersonals. Ohne die Fachkenntnis des OP-Personals (Ärzte und OP-Schwestern) ist eine sinnvolle Neustrukturierung der OP-Logistik nicht durchführbar und würde weit reichende negative Folgen für die Patientenversorgung haben.

Eine Optimierung der OP-Logistik ohne medizinischen Sachverstand führt zu einer mangelhaften Qualität bei der Patientenversorgung.

Die Standardisierung ist die Grundvoraussetzung jeglicher moderner Konzepte. Die Frage, ob standardisiert werden kann, ist durch statistische Erhebungen in Krankenhäusern beantwortet, da heute schon eine Planungssicherheit von über $80 \%$ erreicht werden kann [4]. Zusammen mit dem OP-Personal kann unter Gewährleistung der Versorgungssicherheit der Standardisierungsgrad oder das Standardisierungspotenzial verschiedener Operationen bestimmt werden.

Umgesetzte Konzepte für den Operationssaal sehen eine Kombination aus eingriffsbezogener Versorgung (Fallwagensystem) und Kanban-Methode ${ }^{4}$ (Modulversorgung) vor.

Das Fallwagenkonzept im OP ermöglicht, alle für die Operation benötigten Materialien vor der Operation zusammenzustellen.

Beim Fallwagenkonzept kommt der zeitlichen und örtlichen Trennung von der eigentlichen Operation ein großer Stellenwert zu. Die Vorbereitungen dafür können unabhängig vom operativen Eingriff oder Entsorgung oder Reinigung be-

${ }^{4}$ Die 1953 in Japan entwickelte und dann zuerst bei Toyota eingeführte Methode beschreibt, dass nur angeforderte Ware (damals auf speziellen Karten - „Kanban“ auf japanisch) produziert und geliefert werden darf 

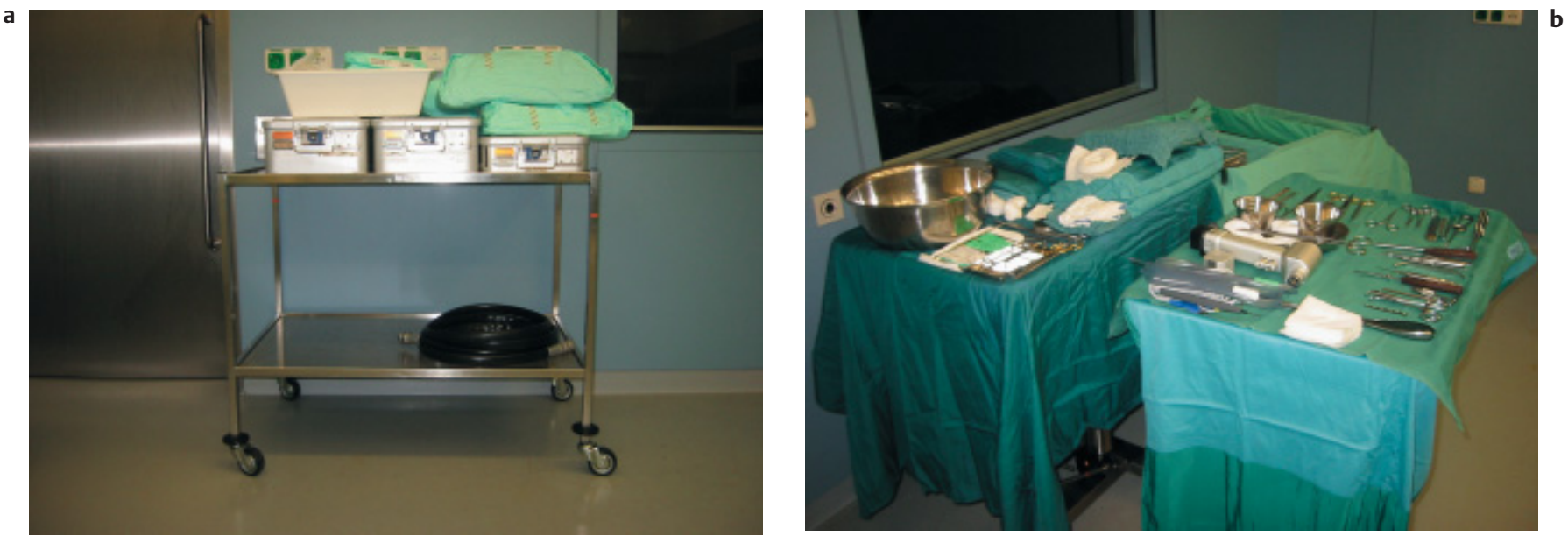

Abb.1 Fallwagen zur Versorgung einer Sprunggelenksfraktur (a) nach der Zusammenstellung und (b) nach Abschluss der Vorbereitung.
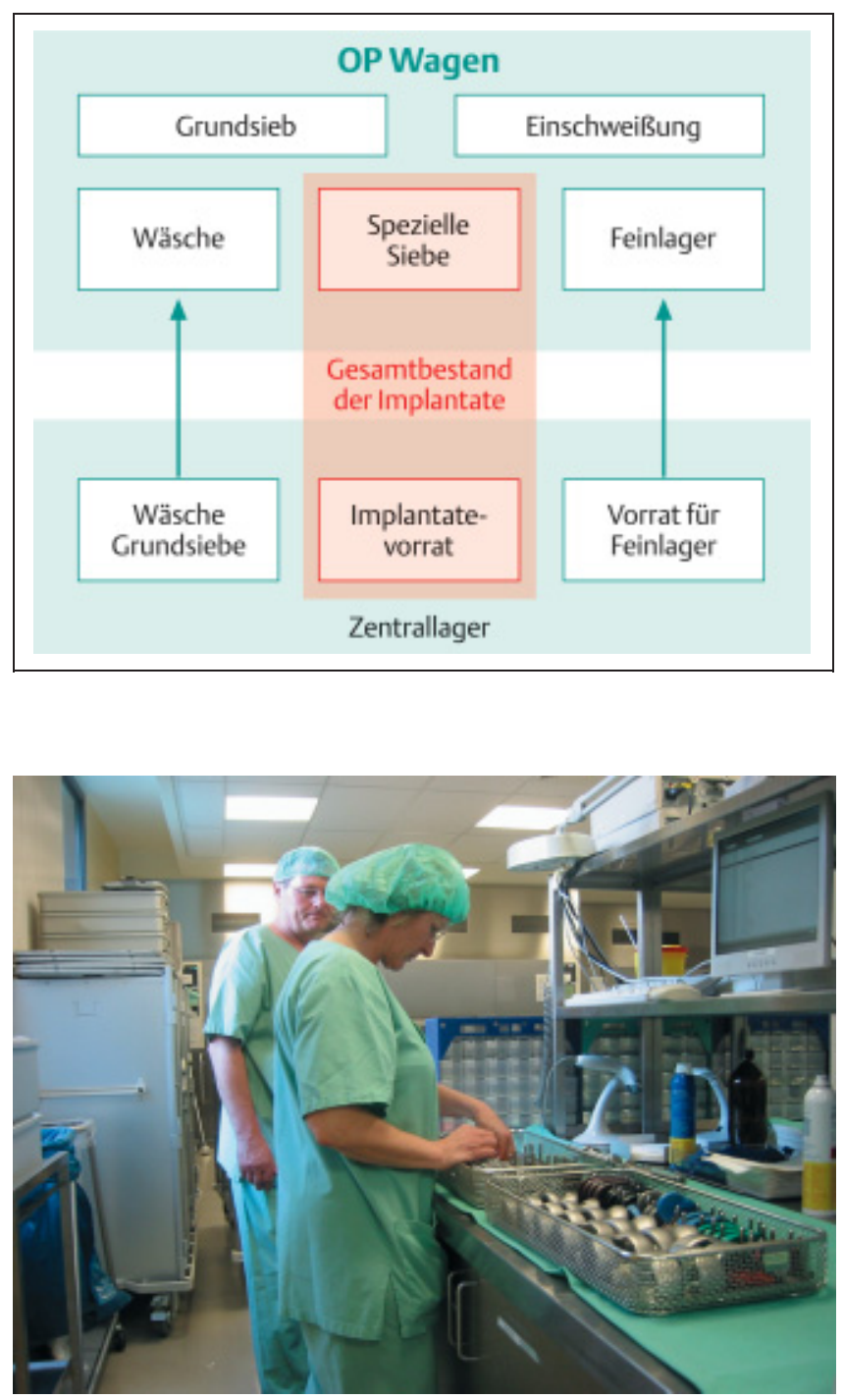

Abb. 2 Die auf dem Fallwagen enthaltenen Artikel kommen aus verschiedenen Lagern. Zu beachten ist, dass sich der Gesamtbestand der Implantate im Haus aus den Implantaten auf den Sieben und den Implantaten im Implantatlager zusammensetzt (gekennzeichnete Fläche).

Abb. 3 Zusammenstellung der Siebe am entsprechenden Arbeitsplatz nach Prüfung auf Sauberkeit und Funktionstüchtigkeit, die elektronisch gespeicherte Packliste ist am Monitor sichtbar. gonnen werden. Die Zusammenstellung des Fallwagens ist eine Kombination verschiedener Zuführungen, zum Beispiel aus dem Zentrallager, der Sterilisation oder den Modulschränken.

Hierbei handelt es sich um verschiedene eingriffsbezogene Bereitstellungen.

- Instrumente (Grundsieb mit allgemeinen Instrumenten) ergänzt durch separat „eingeschweißte“ Instrumente

- Implantate (Spezialsieb mit Implantaten und den dazugehörigen Instrumenten)

- Wäsche (Mantelset und Körperregion abhängiges Abdeckungsset)

- Unspezifische Verbrauchsmaterialien (aus Feinlager)

Siebe und Instrumente, die anhand der OP-Standard - Stückliste bereitgestellt werden, werden auftragsspezifisch durch Aufbereitung (Zentralsterilisation) konfektioniert.

Auch hier werden Standardisierungsmöglichkeiten (allgemeine Grundsiebe und Spezialsiebe) genutzt. Für die Überprüfung der Vollständigkeit der verschiedenen Siebe sind verschiedene Möglichkeiten vorstellbar. Die Zuständigkeit kann im OP verbleiben, in diesem Fall würden fehlerhafte Instrumente und verbrauchte Osteosynthesematerialien aus einem gut erreichbaren Lager sofort ersetzt und das benutzte, aber vollständig durch die OP-Schwester aufgefüllte Sieb in die Sterilisation gegeben werden.

Diese Komplettierung der Siebe ist natürlich auch aus dem hoch qualifizierten Bereich auslagerbar. In der Praxis bedeutet diese Verlegung der Zuständigkeit, dass 

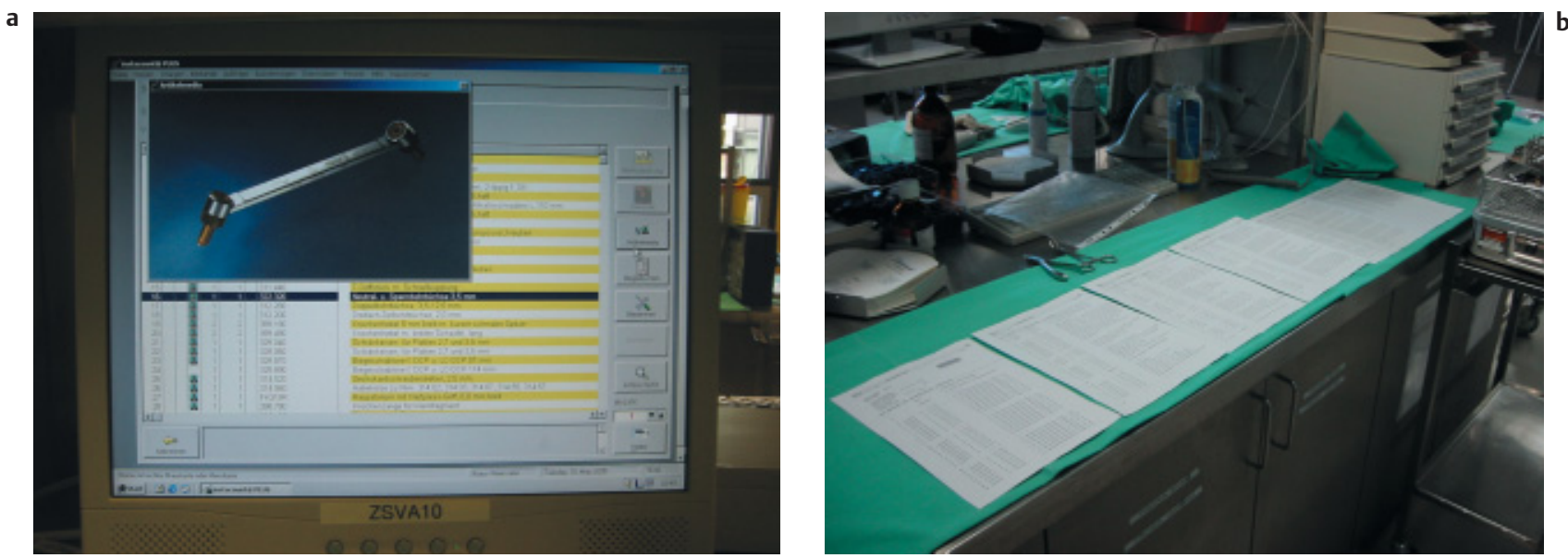

Abb. 4 Zusammenstellung der Siebe anhand von Packlisten. Diese Packlisten sind im (a) Computer hinterlegt, aus Sicherheitsgründen gibt es zusätzlich eine schriftliche Kopie. (b) Für das Sieb „KFI“ umfasst die Packliste 8 Seiten.

die Vollständigkeit der Siebe im Zentrallager anhand einer Packliste hergestellt wird.

Diese Arbeit ist, mit der notwendigen Sorgfalt durchgeführt, auch ohne medizinische Kenntnis durchführbar. Eine denkbare Schwachstelle dieses Systems ist, dass das dafür verantwortliche Personal, trotz hoher Motivation, aufgrund fehlender Sachkenntnis nicht die möglichen gravierenden Folgen auch kleinster Mängel erahnt. Hier hat es sich in der Praxis bewährt, das angesprochene Personal zu Hospitationszwecken in den OP einzuladen, um die Wichtigkeit eines jeden noch so winzigen Details zu erkennen.

Abdeckmaterialien werden auftragsspezifisch von der Logistik und unspezifische Verbrauchsmaterialien aus einem eingriffsnahen Lager (Feinlager) bereitgestellt, welches täglich aus dem Zentrallager beliefert wird.

Zusammen mit dem beschriebenen Fallwagenkonzept wird in einigen Bereichen die so genannte Modulversorgung genutzt. Über diese Modulversorgung erfolgt das Auffüllen der Verbrauchsmaterialien im Zentrallager (verbrauchte Implantate) oder der verbrauchten Kleinmaterialien in Operationsnebenräumen (Feinlager). Diese Modulversorgung basiert auf dem so genannten „pull“-Prinzip, das immer etwas „herangezogen“ werden kann, wenn es benötigt wird. Diese durch Aufträge gesteuerte Lieferung orientiert sich damit sehr am aktuellen Bedarf. Der Vorteil ist eine kleine Lagerfläche, die Versorgungssicherheit muss aber durch eine hohe Lieferzuverlässigkeit gewährleistet werden. Das dem OP-Saal angegliederte Feinlager wird dann zum Beispiel täglich durch das Zentrallager aufgefüllt (hier wird der Lagerplatz im Haus verschoben), das Zentrallager wird von den Lieferanten beschickt (hier wird der Lagerplatz nach außerhalb verschoben).

Die Bestückung des Feinlagers erfolgt täglich und unkompliziert und ersetzt den tatsächlichen Verbrauch. Der externen Lieferung der Verbrauchsmaterialien müssen, da durch komplexere Vorgänge (Bestellung, Lieferkosten, Kontrolle, Stornierung, Rechnungslegung...) gekennzeichnet, wichtige und bedarfsgerechte Analysen vorausgehen. Nur wenn z.B. bei den Osteosynthesematerialien der Verbrauch, eine benötige Reserve, der Bestand und die Lieferzuverlässigkeiten und die Lieferzeiten definiert sind, können die für die Bestellung im Modulsystem wichtigen Größen festgelegt werden:

- der Meldebestand

- der Soll-Bestand

- der Ist-Bestand (Lagerbestand und Bestand der Spezialsiebe)

Der Meldebestand legt die Menge fest, bei der der Bestellvorgang ausgelöst wird, der Soll-Bestand ist die Menge, die nach Lieferung erreicht ist. Meldeund Soll-Bestand können nicht allein statistisch festgelegt werden, auch hier gelten die oben genannten Überlegungen. Nach statistischer Auswertung ist der medizinische Sachverstand unabdingbar. Bei welchen Implantaten ist ein geringerer Meldebestand tolerabel? Welche Implantate sind bei Lieferverzögerungen durch andere Implantate ersetzbar? Ist, um bei unserer Sprunggelenksfraktur zu bleiben, eine Drittelrohrplatte ohne Pro- bleme durch eine winkelstabile Drittelrohrplatte ersetzbar? Falls eine gewünschte 5-Loch-Platte nicht zur Verfügung steht, kann man ohne Bedenken eine 6-Loch-Platte wählen oder vielleicht auch eine 4-Loch-Platte? Müssen immer alle Schrauben mehrfach in der gleichen Länge vorhanden sein oder kann man, in Abhängigkeit von der Lokalisation, eine Schraube wählen, die um nur $2 \mathrm{~mm}$ abweicht?

Alle diese Fragen, die fest mit der gebotenen Produktvielfalt und -menge verknüpft sind, können nur vom qualifizierten Fachpersonal beantwortet werden und dürfen nie zulasten der medizinischen Versorgung auf betriebswirtschaftlicher Ebene entschieden werden.

Die Festlegung der Anzahl der Implantate und der Melde- und Sollbestände kann nur in Abstimmung zwischen Operateuren, OP-Schwestern und Betriebswirten erfolgen.

Wenn die oben genannten organisatorischen Voraussetzungen implementiert sind, ist der technische Ablauf dieser Modulversorgung einfach. Die Artikel sind mit Barcodes versehen und werden bei Ein- und Ausgang gescannt.

Fällt die Menge unter die oben beschriebene definierte kritische Größe (Meldebestand), wird automatisch ein Bestellvorgang ausgelöst. Obwohl dieser erste elektronisch automatisierte Schritt die Prozesse am Beginn der Bestellungskette erheblich vereinfacht, sind die nachfolgenden Schritte wieder personal- und zeitintensiv: Die Bestellungen treffen im Zentraleinkauf ein, werden dort auf 

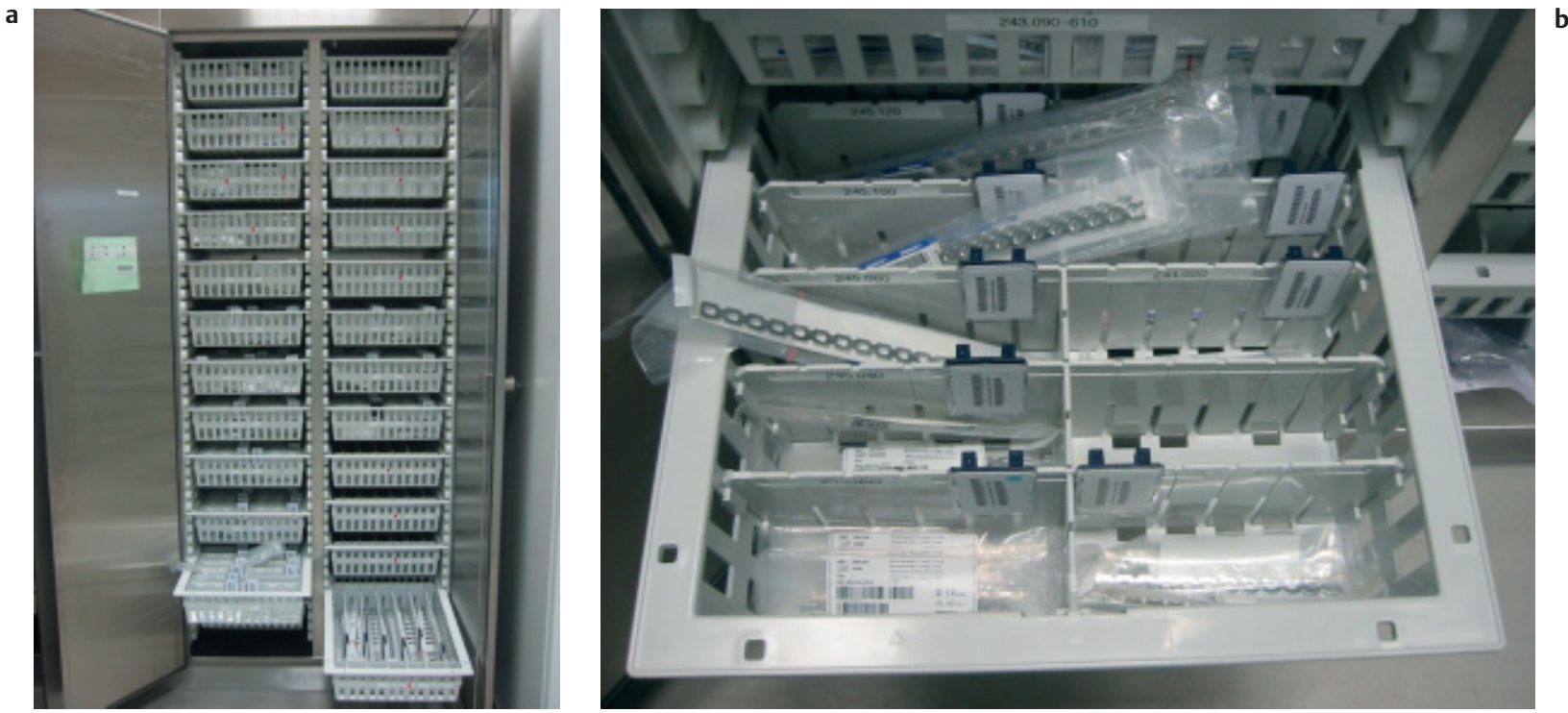

Abb.5 (a) Modulschrank mit Implantaten. Die Artikel sind mit (b) Barcodes versehen und werden bei Ein- und Ausgang gescannt.

einem Bestellformular ausgedruckt und dann gefaxt, die zurüickgesendeten Auftragsbestätigungen werden bearbeitet, der Wareneingang muss gebucht und die Rechnungen müssen dann Kostenstellen zugeordnet und archiviert werden.

Eine im DRG-Zeitalter gebotene Optimierung des Bestellwesens wird zukünftig nur über eine vollständig elektronische Beschaffung umsetzbar sein. Eine vollständig elektronische Beschaffung (EProcurement) lässt sich nicht auf einen elektronischen Bestellvorgang reduzieren, sondern ist gekennzeichnet durch eine Automatisierung des gesamten $\mathrm{Ge}$ schäftsprozesses bis zum elektronischen Lieferschein und zur elektronischen Rechnung. Die Probleme der Einführung der elektronischen Beschaffung sind fehlende Systemstandards. Um eine elektronische Integration von Klinik und Lieferanten/Herstellern zu ermöglichen, sind Investitionen in gleiche elektronische Bestellsysteme (Transaktionsplattformen) erforderlich. Für den Hersteller/Lieferanten bedeutet das, sich auf eine Transaktionsplattform festzulegen und damit (auch wegen der nötigen Investition) eine Bindung an die Klinik. Für die Klinik ist das Erreichen einer kritischen Menge an Herstellern zum Start für die Amortisation der eigenen Investitionen notwendig. Hier spielt das Vertrauen auf eine für beide Seiten lohnende längere Zusammenarbeit mit der Absicherung über lange Vertragslaufzeiten eine große Rolle.

\section{Schlussfolgerung}

Für eine Optimierung der OP-Logistik mit Lagerhaltung und Bestellwesen ist eine genaue Analyse des Bestandes, des Einkaufverhaltens und der Lagermenge notwendig. Weiterhin muss eine korrekte Bedarfsermittlung der benötigten Waren erfolgen, der danach zu definierende Bedarf mit seinen Kenngrößen Melde-, Sollund Ist-Bestand darf nur nach Abstimmung zwischen Operateuren, OP-Schwestern und Betriebswirt festgelegt werden. Eine Standardisierung verschiedenster Bereiche im OP ist für alle Beteiligten wünschenswert und in vieler Hinsicht vorteilhaft; auch hier muss schon in den frühen Planungsphasen das beteiligte Fachpersonal mitbestimmend einbezogen werden. Der logische Schritt der weiteren Optimierung wird zu einer vollständigen elektronischen Beschaffung (E-Procurement) führen, hier sind zukünftig größere positive Effekte zu erwarten.

\section{Literatur}

1 Drauschke S. Revolution im Einkauf - auch im Gesundheitswesen? In: Drauschke, S. Pieper, U. (Hrsg.): Beschaffungslogistik und Einkauf im Gesundheitswesen. Neuwied und Kriftel 2002

2 Large R. Strategisches Beschaffungsmanagement. Wiesbaden 2000

3 Barth A, Lercher H. Projekt „Verbrauchsets im OP“, Methodenentwicklung, Implementierung und Erfahrungen. http://www.oegkv.at/4-2/bildu/bt04/do/bt04-lercher-

barth.pdf

${ }^{4}$ Emmermann M, Giebe T. (R)Evolution im OP. Logistik - DVZ Nr. 126 (2003)

\section{Dr. med. Matthias Müiller}

Assistenzarzt

Dr. med. Andreas Luther

Oberarzt

\section{Petra Ertmer}

Op-Fachschwester

Prof. Dr. med. Karl Heinrich Winker Chefarzt

Klinik für Unfall-, Hand- und Wiederherstellungschirurgie HELIOS Klinikum Erfurt

Nordhäuser Str. 74

D-99089 Erfurt 